\title{
Numerical Study on Propagative Waves in a Periodically Supported Rail Using Periodic Structure Theory
}

\author{
Xi Sheng $\mathbb{D},{ }^{1}$ Huike Zeng $\mathbb{D}$, ${ }^{1}$ Sara Ying Zhang $\mathbb{D},{ }^{1}$ and Ping Wang $\mathbb{D}^{2}$ \\ ${ }^{1}$ Institute of Urban Smart Transportation and Safety Maintenance, Shenzhen University, Shenzhen 518060, China \\ ${ }^{2}$ MOE Key Laboratory of High-Speed Railway Engineering, Southwest Jiaotong University, Chengdu 610031, China
}

Correspondence should be addressed to Sara Ying Zhang; sara.zhangying@szu.edu.cn

Received 21 December 2020; Revised 22 February 2021; Accepted 31 August 2021; Published 14 October 2021

Academic Editor: Steven Chien

Copyright $\left({ }_{0} 2021\right.$ Xi Sheng et al. This is an open access article distributed under the Creative Commons Attribution License, which permits unrestricted use, distribution, and reproduction in any medium, provided the original work is properly cited.

\begin{abstract}
This paper presents the numerical study on propagative waves in a periodically supported rail below $6000 \mathrm{~Hz}$. A periodic rail model, which considers the effects of both the periodic supports and the rail cross section deformation, has been established based on the periodic structure theory and the finite element method. Two selection approaches are proposed to obtain the concerned dispersion curves from the original calculation results of dispersion relations. The differences between the dispersion curves of different support conditions are studied. The propagative waves corresponding to the dispersion curves are identified by the wave modes. The influences of periodic supports on wave modes in pass bands are revealed. Further, the stop band behaviors are investigated in terms of the bounding frequencies, the standing wave characteristics, and the cross-sectional modes. The results show that eight propagative waves with distinct modes exist in a periodically supported rail below $6000 \mathrm{~Hz}$. The differences between the dispersion curves of periodically and continuously supported rails are not obvious, apart from the stop band behaviors. All the bounding-frequency modes of the stop bands are associated with the standing waves. Two bounding-frequency modes of the same stop band can be regarded as two identical standing waves with the longitudinal translation of the quarterwavelength, one of which is the so-called pinned-pinned resonance.
\end{abstract}

\section{Introduction}

The high-frequency rail behaviors play a significant part in the generation of railway rolling noise. It also significantly contributes to the generation of rail corrugation via the dynamic interaction with the wheels $[1,2]$. Investigation of rail vibration behavior is crucial to elucidate these matters. An accurate model for the rail dynamics should take the cross-sectional deformation, waveguide structure, and periodic supports into account. In addition, the effective frequency range for the prediction should extend up to at least $5000 \mathrm{~Hz}[3]$.

The continuously welded rail can be seen as infinitely long. The models which truncate the rail at a particular length will artificially introduce modal behaviors. Besides, the rail is a waveguide, that is, a structure which has a uniform cross section and extends in the longitudinal direction. Thus, its motion is not composed of normal vibration modes but a series of guided waves [4]. A complex wavenumber can be used to describe the velocity and phase of the wave through the real part and the amplitude decay through the imaginary part.

Several numerical methods have been adopted to investigate the guided waves in rails. Ryue et al. [5] established a finite element (FE) model of a short length of the rail with symmetric or antisymmetric boundary conditions at both ends of its length. The modal analysis results were then used to obtain the dispersion relations, group velocities, and mode shapes. An alternative numerical method, known as the guided wave-based finite element method, has been widely employed. This method is also referred to as the semianalytical FE method [6], the waveguide FE method [7], and the wavenumber FE method [5]. In this method, the two-dimensional FE approach is used to discretize the cross section of the waveguide, while a wave solution is assumed in the longitudinal direction. Therefore, it has great advantages 
in modeling wave propagation of waveguides, such as rails. Initial relevant works were done by [8] to obtain the dispersion relations and cross-sectional mode shapes of propagative waves in a free undamped rail. Ryue et al. [5] investigated the waves propagating in the continuously supported rail for frequencies up to $80 \mathrm{kHz}$. Furthermore, $\mathrm{Li}$ et al. [9] improved the rail model by allowing multiple layers of the support to be considered in the modeling and then obtained the dispersion relations of waves. The rail supports, such as rail pads, sleepers, and ballast, are treated as a continuous layer of equivalent springs connected to the rail foot.

The straight railway track structure consists of the same units placed repeatedly along the longitudinal direction of the railway line. The perfect railway track can be seen as a one-dimensional periodic structure. Therefore, the periodic structure theory has also been introduced to investigate the wave propagation. The periodic rail support is the essential characteristic of the standard track structure, which has gained much attention. Tassilly [10] analyzed the propagation of bending waves in a periodically supported rail whose deflection was described by a differential equation of the fourth order. Predictions were made for a rail of a typical European railway track. Wang et al. [11] modeled the rail as a periodically supported Timoshenko beam considering the bending-torsion coupling. The dispersion relations of waves were calculated according to the transfer matrix method and Bloch's theorem.

One of the main effects of periodic supports is the occurrence of the pinned-pinned frequencies, where the wavelengths of different orders are closely related to the fastener spacing [12]. The pinned-pinned frequency has been associated with some forms of rail corrugation, which leads to the problems of noise and track structure damage [13]. Moreover, existing researches show that the dispersion relations of waves propagating in a periodic structure exhibit stop and pass band behaviors [14-16]. The stop bands (also called band gaps) are the frequency ranges between the dispersion curves, where wave propagation is prohibited. The other frequency ranges are called pass bands, where the dispersion curves exist and waves can propagate freely. Reference [11] used beam models to explore the stop and pass band behaviors of waves propagating in the periodically supported rail.

Although many studies as previously described focused on the wave propagation in the rail, few attempted to put insight into the effects of both the periodic supports and the rail cross-section deformation. These two factors are the key to an accurate prediction of wave propagation for rails in a real state. However, it is difficult to include the periodic support in the guided wave-based finite element method due to the model assumption, while the rail cross-section deformation cannot be considered in the periodically supported beam model. Beam models are not accurate at high frequencies since the cross section of the rail deforms significantly above $1500 \mathrm{~Hz}$ [17]. As the FE method has the advantage of calculating rail cross-section deformation and the periodic structure theory is particularly useful for periodically supported rails, they are both utilized to study the propagative waves in a periodically supported rail.

In this paper, a periodic rail model has been established, which considers the effects of both the periodic supports and the rail cross-section deformation. Two selection approaches are proposed to obtain the concerned dispersion curves from the original calculation results of dispersion relations. The differences between the dispersion curves of different support conditions are studied. The propagative waves corresponding to the dispersion curves are identified by the wave modes. The influences of periodic supports on wave modes in pass bands are revealed. Further, the stop band behaviors are investigated in terms of the bounding frequencies, standing wave characteristics, and cross-sectional modes.

\section{Modeling of a Periodically Supported Rail}

2.1. Unit Cell. The periodic rail model is established in this section, as shown in Figure 1. A short rail and a rail pad form the model which can be seen as a unit cell of the infinitely long and periodically supported rail.

A CHN60 rail considered as the isotropic elastic material is modeled with three-dimensional solid elements. The length of the model is equal to the fastener spacing. A rail pad is laid beneath the middle of the model, which is modeled with multiple discrete linear springs. The upper nodes of the springs are connected to the rail foot, while the lower nodes are fixed. These springs are modeled homogeneously in the rectangular rail pad area, whose width is equal to that of the bottom of the rail foot. The rail pad inflicts constraints on the rail in three directions, that is, vertical, longitudinal, and lateral stiffnesses. The damping is not considered in the model. The parameter values are listed in Table 1.

2.2. Periodic Boundary Condition. When an ideal elastic medium (continuous, homogeneous, isotropic, and perfectly elastic) deforms slightly, the governing equation of motion without body force can be expressed as follows [18]:

$$
\rho \frac{\partial^{2} \mathbf{u}(\mathbf{r}, t)}{\partial t^{2}}=(\lambda+\mu) \nabla \nabla \cdot \mathbf{u}(\mathbf{r}, t)+\mu \nabla^{2} \mathbf{u}(\mathbf{r}, t),
$$

where $\boldsymbol{u}(\boldsymbol{r}, t)$ is the displacement field, $\boldsymbol{r}$ is the coordinate vector, $t$ is the time, $\rho$ is the density, $\lambda$ and $\mu$ are the Lamé constants, $\nabla$ is the Hamilton differential operator, and $\bullet$ is the inner product. According to Bloch theorem, the solution of equation (1) for a periodic structure can be expressed as follows [19]:

$$
\mathbf{u}(\mathbf{r})=\mathrm{e}^{\mathrm{i} \mathbf{K} \cdot \mathbf{r}} \mathbf{u}_{\mathbf{K}}(\mathbf{r})
$$

where $\boldsymbol{K}$ is the wave vector in the reciprocal space and $\boldsymbol{u}_{\boldsymbol{K}}(\boldsymbol{r})$ is a periodical function with the same periodicity as the unit cell. The periodicity can be expressed as follows:

$$
\mathbf{u}_{\mathbf{K}}(\mathbf{r}+\mathbf{a})=\mathbf{u}_{\mathbf{K}}(\mathbf{r})
$$



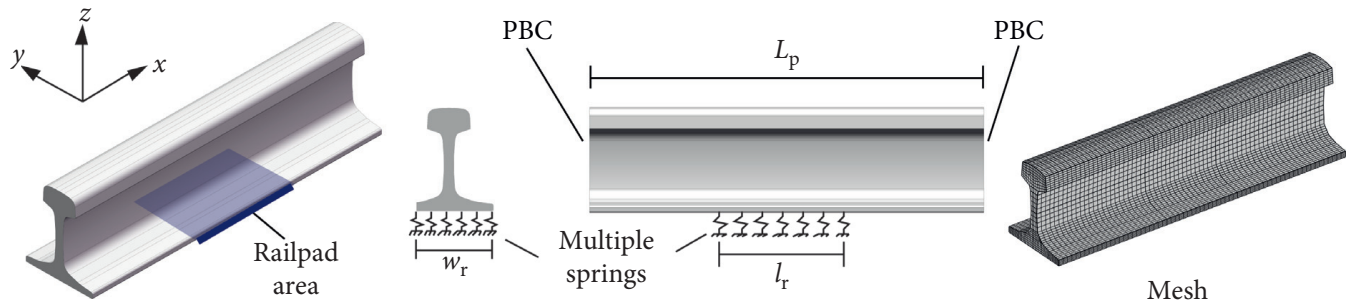

Figure 1: The periodic rail model.

TABLe 1: Model parameters.

\begin{tabular}{lcc}
\hline Track component & Parameter & Value \\
\hline \multirow{4}{*}{ CHN60 rail } & Young's modulus & $E=210 \mathrm{GPa}$ \\
& Poisson's ratio & $\nu=0.3$ \\
& Density & $\rho=7830 \mathrm{~kg} / \mathrm{m}^{3}$ \\
& Length & $L=0.65 \mathrm{~m}$ \\
\hline Width & $w_{r}=0.15 \mathrm{~m}$ \\
Rail pad & Length & $l_{\mathrm{r}}=0.16 \mathrm{~m}$ \\
& Vertical stiffness & $k_{\mathrm{v}}=70 \mathrm{kN} / \mathrm{mm}$ \\
& Longitudinal stiffness & $k_{\mathrm{l}}=20 \mathrm{kN} / \mathrm{mm}$ \\
& Lateral stiffness & $k_{\mathrm{L}}=30 \mathrm{kN} / \mathrm{mm}$ \\
& Fastener spacing & $d=0.65 \mathrm{~m}$ \\
\hline
\end{tabular}

where $\boldsymbol{a}$ is the periodic constant vector. Substituting equation (3) into equation (2) yields the periodic boundary condition:

$$
\mathbf{u}(\mathbf{r}+\mathbf{a})=\mathrm{e}^{i \mathbf{K} \cdot(\mathbf{r}+\mathbf{a})} \mathbf{u}_{\mathbf{K}}(\mathbf{r}+\mathbf{a})=\mathrm{e}^{i \mathbf{K} \cdot \mathbf{a}} \mathrm{e}^{i \mathbf{K} \cdot \mathbf{r}} \mathbf{u}_{\mathbf{K}}(\mathbf{r})=\mathrm{e}^{i \mathbf{K} \cdot \mathbf{a}} \mathbf{u}(\mathbf{r}) .
$$

As the model is a one-dimensional periodic structure, the periodic constant vector $\boldsymbol{a}$, the wave vector $\boldsymbol{K}$, and the coordinate vector $\boldsymbol{r}$ can be substituted by the length of the model $L$, the wavenumber $k$, and the longitudinal coordinate $x$, respectively. Then, the periodic boundary condition can be written as follows:

$$
\mathbf{u}(x+L)=\mathrm{e}^{\mathrm{i} k L} \mathbf{u}(x) .
$$

The period boundary condition is set on two sides of the rail. The combination of the periodic boundary condition and the modal analysis gives the eigenvalue problem involving the dispersion relation. Then, the dispersion curves of waves propagating in a periodically supported rail can be obtained by calculating the eigenfrequencies at different wavenumbers, while the corresponding eigenvectors can be used to describe the wave modes. The dispersion curves are complex with damping considered in the model, whereas they will be real if damping is not considered. Since the wavenumber is set artificially prior to solving the eigenvalue problem, it is feasible to set the real wavenumbers rather than complex wavenumbers. Consequently, the damping is not considered in the model, and the focus of this paper is on the propagative waves, which can occur at frequencies above their "cut-on" frequencies.

\subsection{Calculation of Dispersion Relations Using the Finite El-} ement Method. To calculate the dispersion relations of propagative waves in a periodically supported rail, the finite element software is utilized to solve the model. The rail is meshed with eight-node hexahedral solid elements. In the rail pad area, each node of the bottom of the rail foot is connected by a linear three-directional spring element, which represents the constraints of the fastener. To capture the wave features at high frequencies, the in-plane mesh size of the rail cross section is shorter than $5 \mathrm{~mm}$, while the mesh size in the longitudinal direction does not exceed $1 \mathrm{~cm}$. The mesh sizes are proved to be fine enough in the frequency range of interest because the finer meshing gives nearly the same results. The mesh of the periodic rail model is shown in Figure 1.

By letting the wavenumber $k$ sweep the real interval $[-4 \pi / L, 6 \pi / L]$ at regular intervals of $\pi / L / 30$ and then calculating the corresponding eigenfrequencies, we can obtain the original dispersion relation results, as shown in Figure 2.

2.4. Selection of the Concerned Dispersion Curves. Let $f_{n}(k)$ denote the $n$th eigenfrequency when the wavenumber is equal to $k$. From Figure 2, we can find that the original results of dispersion relations have three features. First, the dispersion relations are symmetric with respect to $k=0$, which means $f_{n}(k)=f_{n}(-k)$. Because the model is a periodic and symmetric structure, there exist two propagative waves, which propagate in opposite directions and have the same propagation characteristics. Second, $f_{n}(k)$ is a periodic function with the period $2 \pi / L$. We transform equation (5) into the following:

$$
\mathbf{u}(x+L)=\mathrm{e}^{i k L} \mathbf{u}(x)=\mathrm{e}^{i(k L+2 \pi)} \mathbf{u}(x)=\mathrm{e}^{i(k+2 \pi / L) L} \mathbf{u}(x)=\mathrm{e}^{i k * L} \mathbf{u}(x) \quad k *=k+\frac{2 \pi}{L} .
$$

From equation (6), we can find that the same modal results will be obtained if the wavenumber $k$ is substituted by $k^{*}$. Thus, we can get $f_{n}(k)=f_{n}\left(k^{*}-\right)$; that is, $f_{n}(k)=f_{n}$ $(k+2 \pi / L-)$. Third, $f_{n}(k)$ is symmetric with respect to 


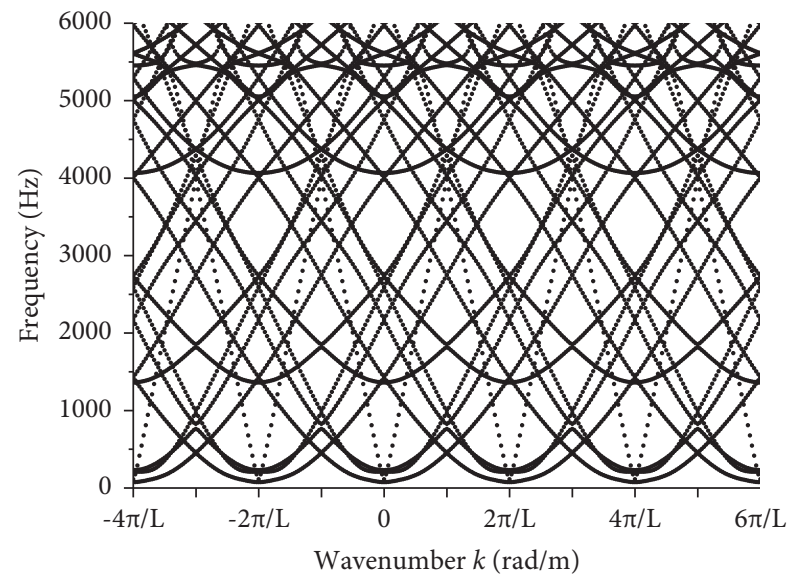

FIGURE 2: The original results of dispersion relations.

$k=m \pi / L$, where $m$ denotes the integer. This feature can be derived from the first two features, shown as follows:

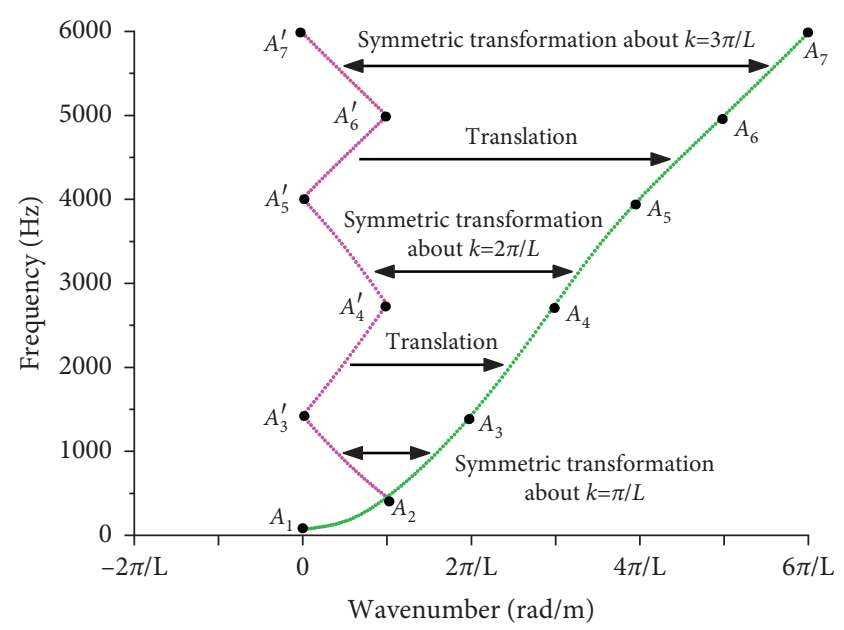

FIgURE 3: Selection of the dispersion curve of wave $A$.

$$
f_{n}(k)=f_{n}(-k) \Rightarrow f_{n}(k)=f_{n}\left(\left( \pm \frac{2 \pi}{L} \pm \ldots \pm \frac{2 \pi}{L}\right)-k\right) \Rightarrow f_{n}(k)=f_{n}\left(m \frac{2 \pi}{L}-k\right) \Rightarrow f_{n}(k)=f_{n}\left(m \frac{\pi}{L}-\left(k-m \frac{\pi}{L}\right)\right)
$$

Based on three features, the whole dispersion relations can be obtained by the translations and symmetric transformations of the part where wavenumbers are in the interval $[0, \pi / L]$. This interval is called the irreducible Brillouin zone of the one-dimensional periodic structure. However, the dispersion relations in Figure 2 include much redundant information. Although all the points are calculated by the mathematical derivation, some dispersion curves are physically unacceptable for this model. For example, the curve where the frequency decreases with the increasing wavenumber indicates the wave having a negative group velocity; that is, the direction of the group velocity is opposite to the propagation direction. In addition, the dispersion curves of the same shape, which can be obtained by the horizontal translations of each other, indicate the same wave due to the periodicity. These phenomena arise from the mathematical mechanism, which need to be removed artificially.

Two approaches are utilized for the selection of the concerned dispersion curves. First, as previously mentioned, the dispersion curves can be obtained by the translations and symmetric transformations of the part within the irreducible Brillouin zone. Second, we can identify the wave modes of different points in the dispersion relations and then select the concerned curves from points where the corresponding wave modes are of the same type. The first approach is illustrated in Figure 3 for the case of wave $A$.

\section{Dispersion Curves and Wave Modes in Pass Bands}

3.1. Dispersion Curves. By applying the selection approaches to Figure 2, we can obtain the dispersion curves of propagative waves in a periodically supported rail below $6000 \mathrm{~Hz}$, as shown in Figure 4.

Eight propagative waves (denoted by $A \sim H$ ) are found in a periodically supported rail below $6000 \mathrm{~Hz}$. To investigate the effect of the periodic supports on the dispersion curves and simultaneously verify the precision of the model, the dispersion curves of continuously supported and free rails are calculated and shown in Figure 5. In the periodic rail model of a continuously supported rail, the rail pad area evenly covers the entire rail foot. The total stiffness of the discrete linear springs in three directions is equivalent to that of a periodically supported rail.

Below $6000 \mathrm{~Hz}$, eight propagative waves are also found in continuously supported and free rails. By comparing the shapes and locations of the dispersion curves, we can find that the results for continuously supported and free rails perfectly match those of existing works done by the guided wave-based finite element method [8], which verifies the precision of the periodic rail model.

Figure 5 reveals that the overall differences between the dispersion curves of three support conditions are small, above $2000 \mathrm{~Hz}$, where the wave motion in the rail has been effectively isolated from the rest of the track structure. From the comparison between the periodically supported and free rails, we can find that the periodic supports have significant effects on waves $A \sim E$ near their cut-on frequencies. However, the overall difference between the dispersion curves of periodically and continuously supported rails is not obvious below $6000 \mathrm{~Hz}$. They almost coincide with each other, even near the cut-on frequencies. 


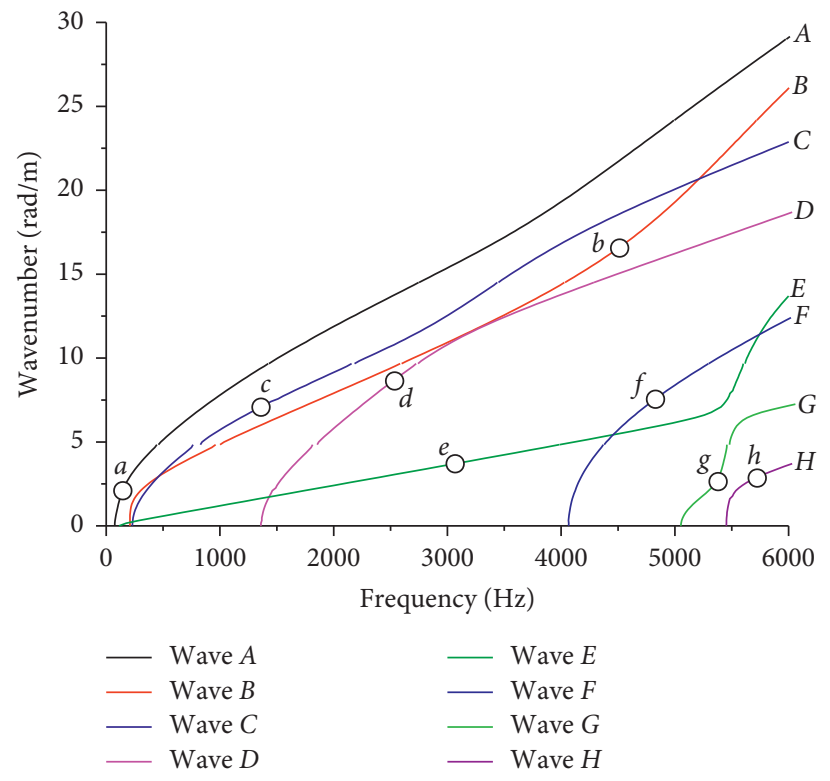

Figure 4: Dispersion curves of propagative waves in a periodically supported rail.

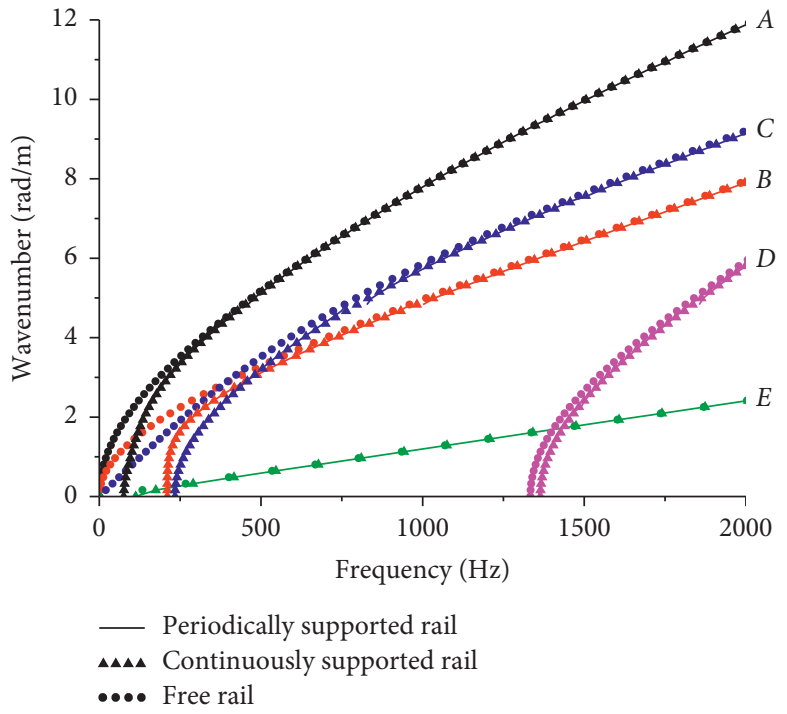

(a)

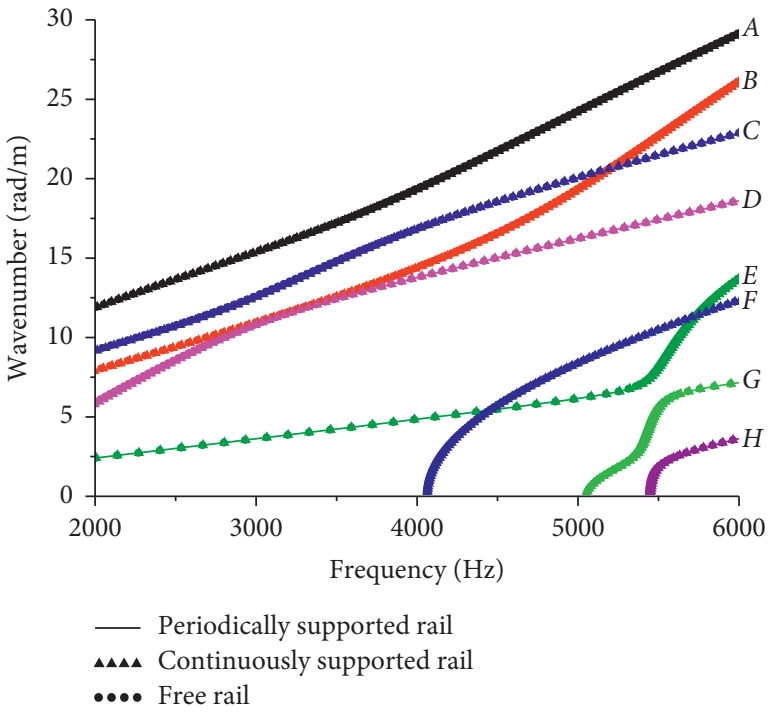

(b)

FIGURE 5: Dispersion curves of different support conditions: (a) 0-2000 Hz; (b) 2000-6000 Hz.

3.2. Wave Modes. To further characterize these waves, the wave modes corresponding to the marked points (denoted by $a \sim h$ ) in Figure 4 are shown in Figure 6, which contains the total displacements of the model, the out-of-plane and inplane displacements of different cross sections, and the front view of the in-plane displacements of the cross section in the middle of the model. The colors indicate the displacement values in the particular phase state. The black lines represent the undeformed profile.

It is noteworthy that the wave modes of a wave vary with the increasing frequency. By scanning the wave modes in the whole frequency range, waves $A \sim H$ can be considered as the lateral bending wave, the vertical bending wave, the torsion wave, the bending-torsion wave, the longitudinal-vertical wave, the bending-torsion wave, the longitudinal-vertical wave, and the vertical-longitudinal wave, respectively.

As the free and continuously supported rails have uniform cross sections and boundary conditions, their modes of all cross sections are the same. The displacement amplitudes of different cross sections are identical, while the values are not same because of the phase difference. However, the periodic supports of fasteners lead to distinct boundary conditions at different positions. To compare the modes of different cross sections for a periodically supported rail, the wave modes with the increasing frequency are shown in Figure 7. The colors indicate the displacement amplitude, which is different to Figure 6. 
(a)

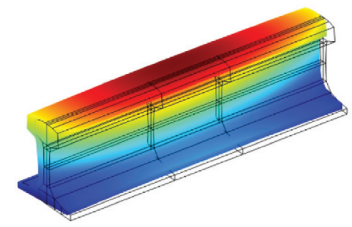

(b)

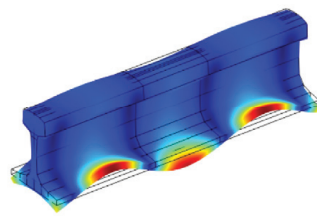

(c)

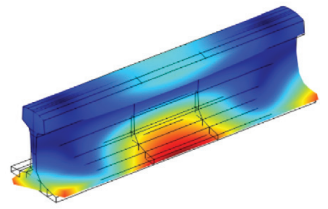

(d)

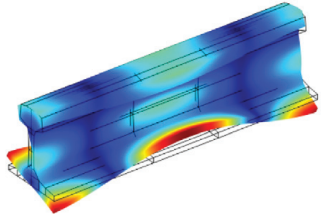

(e)

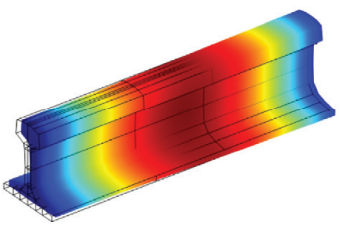

(f)

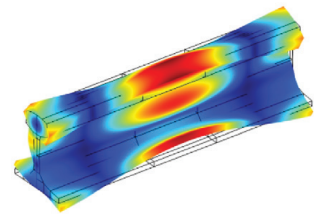

$(\mathrm{g})$

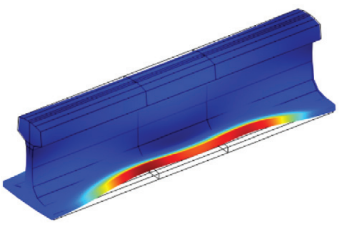

(h)

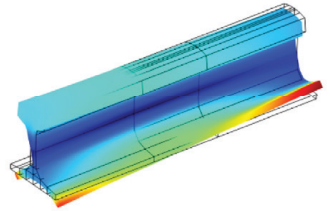

Out-of-plane

displacements
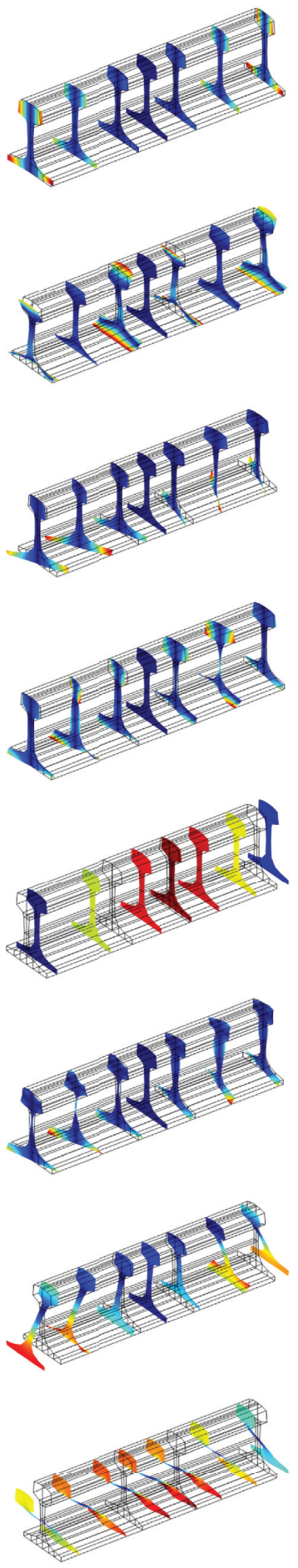

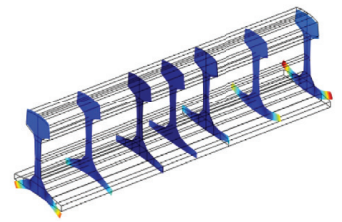

displacements

(front view)
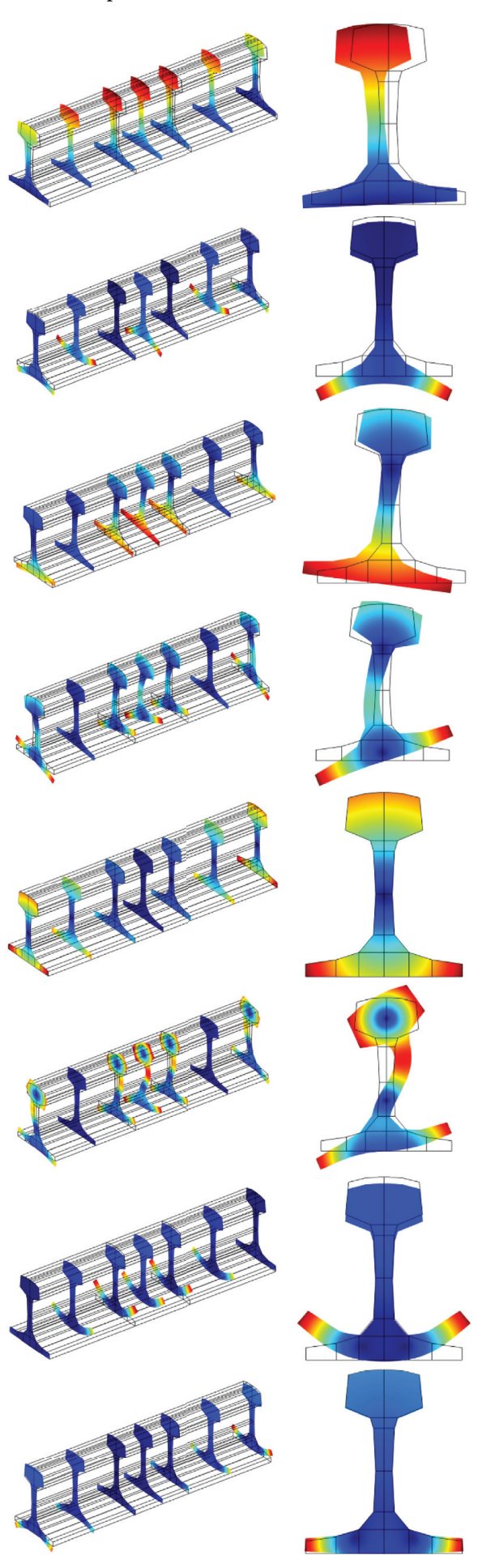

FIGURE 6: Wave modes corresponding to the marked points (a) (h). 


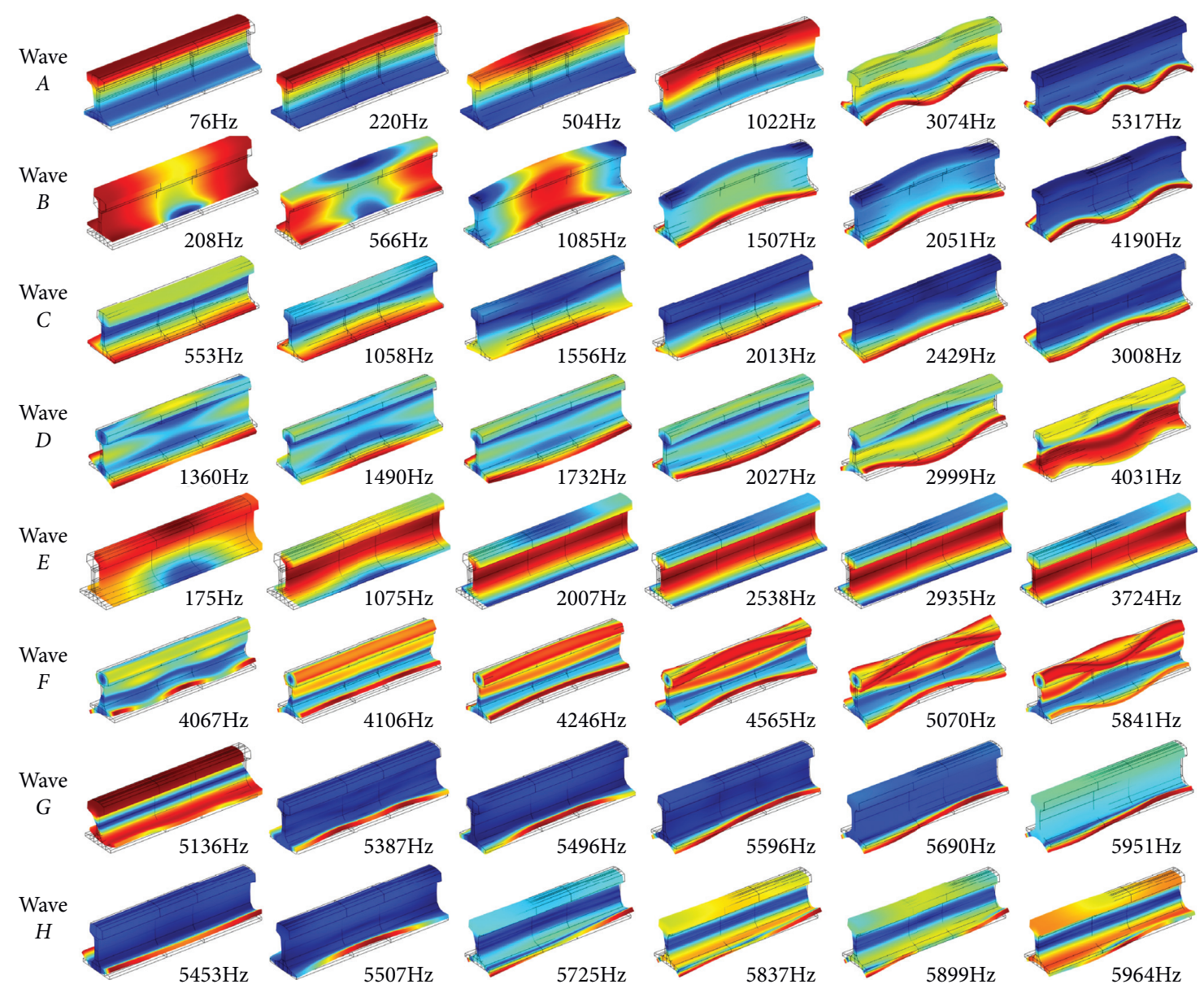

Minimal

displacement amplitude
Maximum

displacement amplitude

FIgURE 7: The wave modes of eight propagative waves.

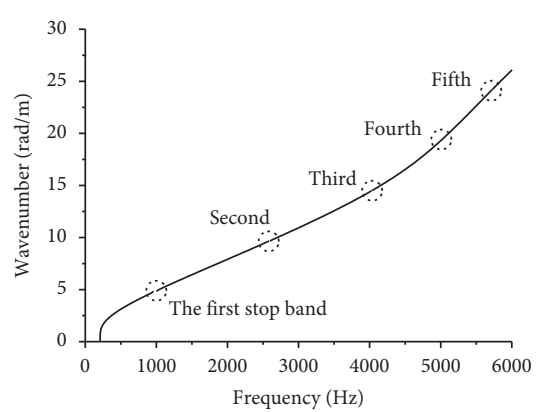

(a)

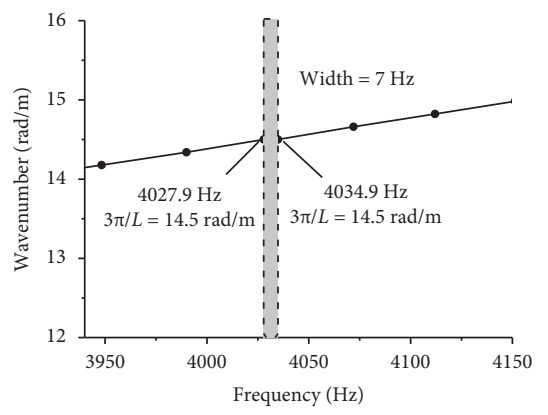

(d)

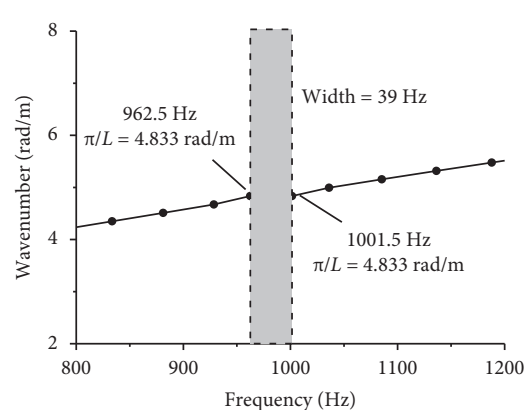

(b)

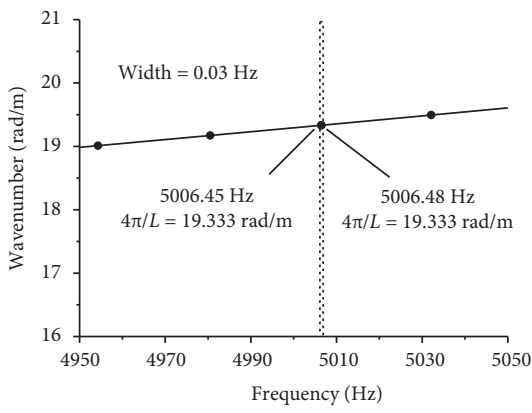

(e)

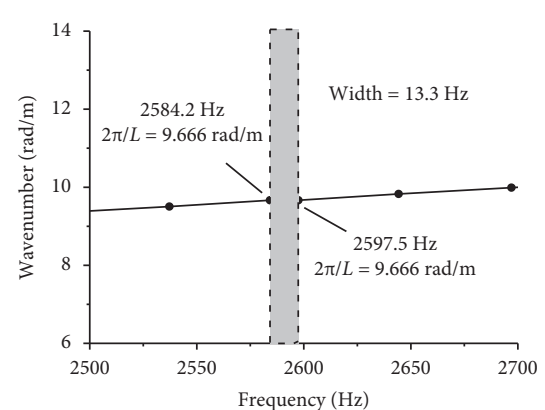

(c)

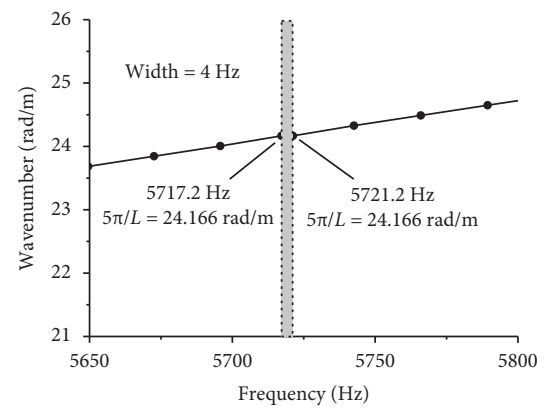

(f)

Figure 8: The dispersion curves and stop bands of the wave $B$ : (a) the dispersion curves; (b-f) the first to fifth stop bands. 
TABLE 2: The bounding frequencies of stop bands.

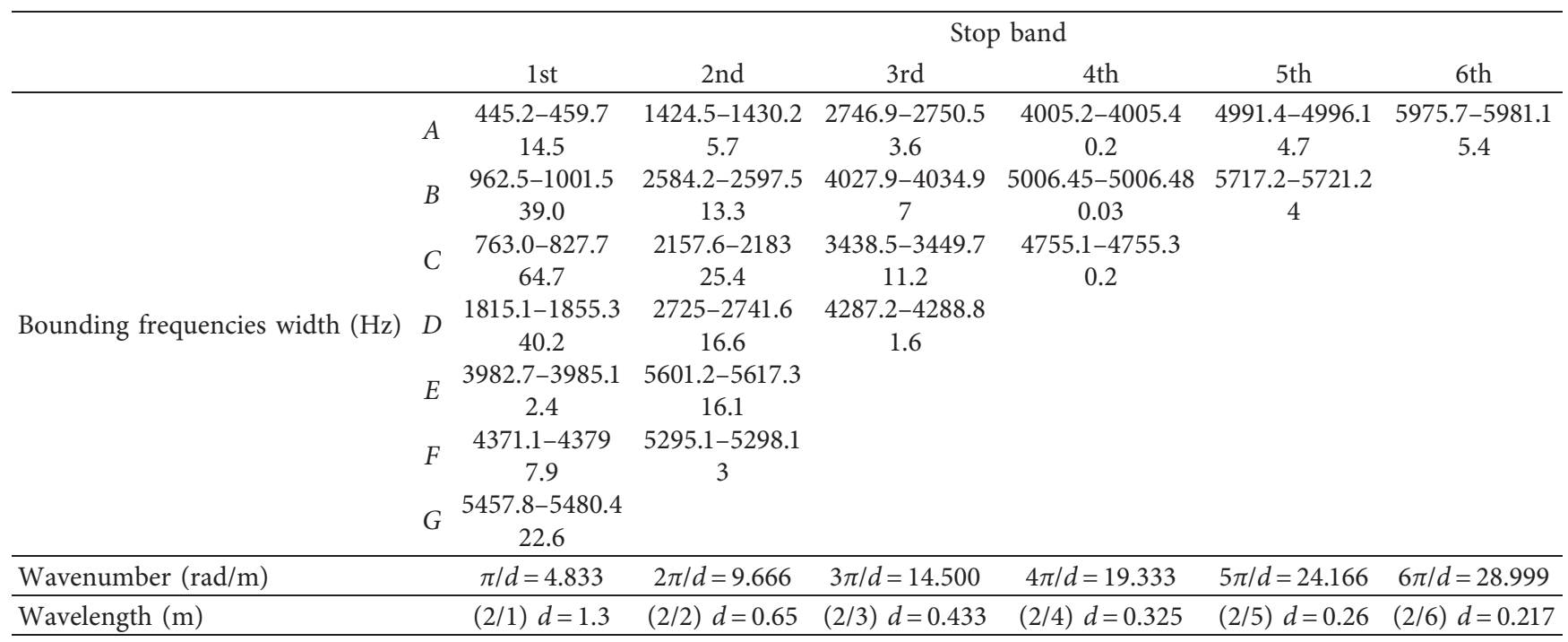

Figure 7 shows that the periodic supports result in the cross-sectional mode differences between the rail pad and non-rail pad areas. The influences of periodic supports on eight propagative waves are as follows: (1) the modes of different cross-sections for wave A are same at low frequencies. The periodic supports have little influence on wave A. (2) The upper bounds of influenced frequency ranges for waves $B \sim G$ are about $1500 \mathrm{~Hz}, 2000 \mathrm{~Hz}, 1700 \mathrm{~Hz}, 2500 \mathrm{~Hz}$, $4100 \mathrm{~Hz}$ and $5600 \mathrm{~Hz}$, respectively. The influences vanish above those frequencies. (3) The cross-sectional modes of rail pad and non-rail pad areas are different below $6000 \mathrm{~Hz}$ for wave $H$.

\section{Stop Band Behaviors}

As can be seen in Figure 4, the dispersion curves of propagative waves in a periodically supported rail are discontinuous. The stop bands alternate with the pass bands. In this section, the stop band behaviors are analyzed in terms of the bounding frequencies, the standing wave characteristics, and the cross-sectional modes.

4.1. The Bounding Frequencies. As the wave $B$ (vertical bending wave) is typical in the track dynamics studies, this section takes it as an illustration to elaborate the boundingfrequency properties. The dispersion curves and stop bands of the wave $B$ are shown in Figure 8 .

Figure 8 reveals that the dispersion relations are discontinuous at $k=n \pi / L$. No curve enters into the frequency ranges of $\quad 962.5-1001.5 \mathrm{~Hz}, \quad 2584.2-2597.5 \mathrm{~Hz}, \quad 4027.9-4034.9 \mathrm{~Hz}$, $5006.45-5006.48 \mathrm{~Hz}$, and $5717.2-5721.2 \mathrm{~Hz}$. These frequency intervals are called the stop bands, and therefore, five stop bands of the wave $B$ exist below $6000 \mathrm{~Hz}$.

The bounding frequencies of waves $A \sim G$ are summarized in Table 2. The wave $H$ has no stop band below $6000 \mathrm{~Hz}$.

Below $6000 \mathrm{~Hz}$, the numbers of the stop bands of waves $A \sim G$ are six, five, four, three, two, two, and one, respectively. For each wave, the wavenumber and the wavelength of the $N$ th stop band are given by $k_{N}=N \pi / L=N \pi / d$ and $\lambda_{N}=2 \pi / k_{N}=(2 / N) d$, respectively; the first stop band has the maximum width compared with the higher-order stop bands.

4.2. The Standing Wave Characteristics. The wave modes at the stop-band bounding frequencies of waves $A \sim G$ are shown in Figure 9. The colors indicate the displacement values in the particular phase state.

All the bounding-frequency modes of the stop bands are associated with the standing waves. The typical cross sections of these standing waves can be divided into two groups. The section group \# 1 includes the sections above the center of the rail pad area and at the $N$-equal-part division points of the span. The section group \#2 includes the sections at the centers of $N$-equal-part segments of the span. These two section groups can be used to locate the cross-sections of nodes and antinodes.

One of two bounding-frequency modes of the same stop band is the so-called pinned-pinned resonance, that is, the modes with the red asterisks at their top-left corners in Figure 9. Either the lower-bounding-frequency mode or the upper-bounding-frequency mode corresponds to the pinned-pinned resonance, which is related to the order of the stop band. Besides, the lower-bounding-frequency mode of the first stop band of the wave $E$ corresponds to the firstorder longitudinal pinned-pinned resonance. The sections of group \#1 have no out-of-plane displacements in this lowerbounding-frequency mode.

Furthermore, we can find that the two bounding-frequency modes of the same stop band can be regarded as two identical standing waves with the longitudinal translation of the quarter-wavelength. Thus, the minimal longitudinal distance between the cross sections of nodes (or antinodes) in two bounding-frequency modes is given by $\lambda_{N} / 4$. Cross sections of nodes and antinodes in the lower-boundingfrequency mode coincide with those of antinodes and nodes in the upper-bounding-frequency mode, respectively. 


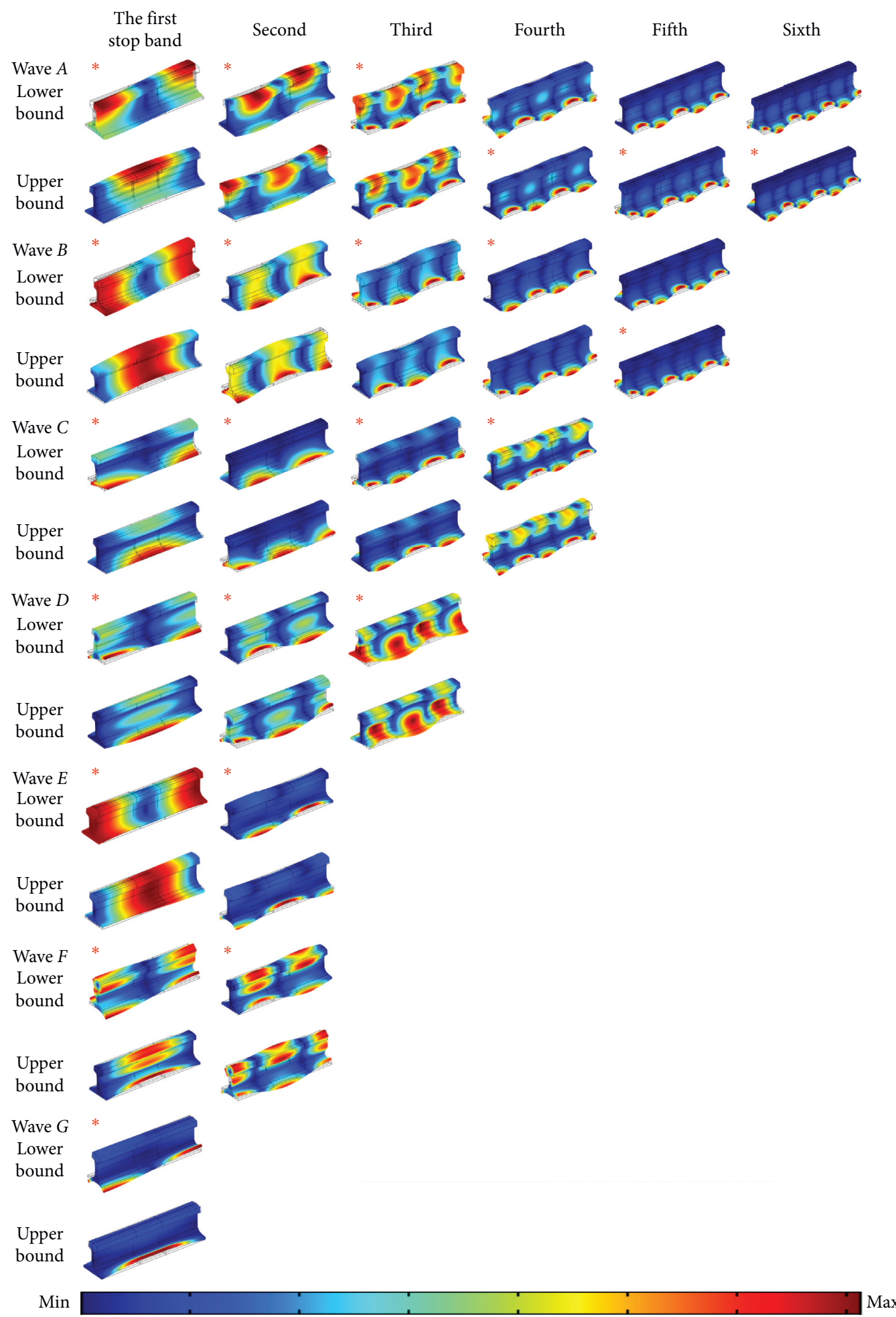

Figure 9: The wave modes at the stop-band bounding frequencies of waves $A \sim G$.

4.3. Cross-Sectional Modes. To elaborate the wave motion and the deformation at different bounding frequencies, the cross-sectional modes of the typical cross sections (i.e., section groups \#1 and \#2) are investigated in terms of inplane and out-of-plane displacements in this section. The cross-sectional modes of propagative waves at the lowerbounding frequencies are shown in Table 3.

With regard to the cross sections of two section groups in a lower-bounding-frequency mode, either in-plane or outof-plane displacements are zeros, but not both. Besides, if 


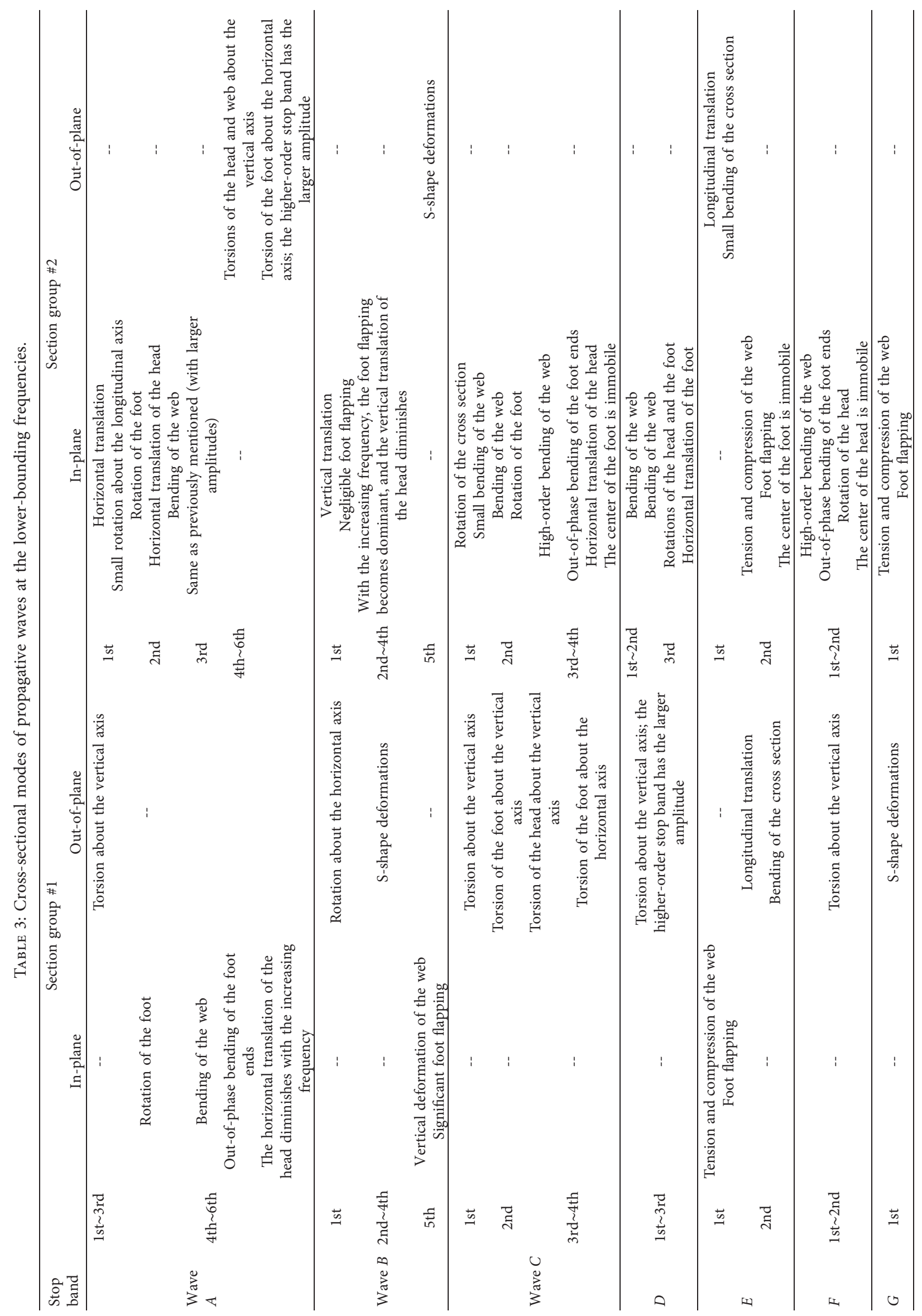


one section group has no in-plane displacement, the other section group must have no out-of-plane displacement, and vice versa. For waves $A, B$, and $E$, the sections without inplane or out-of-plane displacements may come from either section group, which is determined by the order of the stop band.

As previously mentioned, the two bounding-frequency modes of the same stop band can be regarded as two identical standing waves with the longitudinal translation of the quarter-wavelength. For each stop band of waves, the cross-sectional modes of section group \#1 at lower and upper boundary frequencies are identical to those of group \#2 at upper and lower boundary frequencies, respectively. It has been verified by investigating the cross-sectional modes of propagative waves at the upper-bounding frequencies.

\section{Conclusions}

The main conclusions can be drawn as follows:

(1) Eight propagative waves with distinct modes exist in a periodically supported rail below $6000 \mathrm{~Hz}$. The overall differences between the dispersion curves of three support conditions (periodic supports, continuous supports, and no supports) are small, above $2000 \mathrm{~Hz}$. The differences between the dispersion curves of periodically and continuously supported rails are not obvious, apart from the stop band behaviors. However, the periodic supports result in the cross-sectional mode differences between the rail pad and non-rail pad areas.

(2) The stop-band numbers of eight propagative waves are six, five, four, three, two, two, one, and zero, respectively. For each wave, the wavenumber and the wavelength of the $N$ th stop band are given by $N \pi / d$ and $(2 / N) d$, where $d$ is the fastener spacing; the first stop band has the maximum width compared with the higher-order stop bands.

(3) All the bounding-frequency modes of the stop bands are associated with the standing waves. Two bounding-frequency modes of the same stop band can be regarded as two identical standing waves with the longitudinal translation of the quarter-wavelength, one of which is the so-called pinned-pinned resonance.

(4) With regard to typical standing-wave sections in bounding-frequency modes, either in-plane or out-of-plane displacements are zeros, but not both. These typical standing-wave sections can be divided into two groups. If one section group has no in-plane displacement, the other section group must have no out-of-plane displacement, and vice versa.

\section{Data Availability}

The data used to support the findings of this study are available from the corresponding author upon request.

\section{Conflicts of Interest}

The authors declare no potential conflicts of interest with respect to the research, authorship, and/or publication of this article.

\section{Acknowledgments}

The authors disclosed receipt of the following financial support for the research, authorship, and/or publication of this article: the National Natural Science Foundation of China (no. 52008264), Special Fund for Science and Technology Innovation Strategy of Guangdong Province (2020A0505100065), and Shenzhen Science and Technology Program (KQTD20180412181337494).

\section{References}

[1] K. Wei, Y. Dou, F. Wang, P. Niu, P. Wang, and Z. Luo, "Highfrequency random vibration analysis of a high-speed vehicletrack system with the frequency-dependent dynamic properties of rail pads using a hybrid SEM-SM method," Vehicle System Dynamics, vol. 56, no. 12, pp. 1838-1863, 2018.

[2] W. Zhai, P. Liu, J. Lin, and K. Wang, "Experimental investigation on vibration behaviour of a CRH train at speed of 350 $\mathrm{km} / \mathrm{h}$," International Journal of Reality Therapy, vol. 3, no. 1, pp. 1-16, 2015.

[3] D. J. Thompson, Railway Noise and Vibration: Mechanisms, Modelling and Means of Control, Elsevier, Oxford, UK, 2008.

[4] M. Yuan, P. W. Tse, W. Xuan, and X. Wenjin, "Extraction of least-dispersive ultrasonic guided wave mode in rail track based on floquet-bloch theory," Shock and Vibration, vol. 2021, Article ID 6685450, 10 pages, 2021.

[5] J. Ryue, D. J. Thompson, P. R. White, and D. R. Thompson, "Investigations of propagating wave types in railway tracks at high frequencies," Journal of Sound and Vibration, vol. 315, no. 1-2, pp. 157-175, 2008.

[6] T. Hayashi, W.-J. Song, and J. L. Rose, "Guided wave dispersion curves for a bar with an arbitrary cross-section, a rod and rail example," Ultrasonics, vol. 41, no. 3, pp. 175-183, 2003.

[7] P. W. Loveday, "Analysis of piezoelectric ultrasonic transducers attached to waveguides using waveguide finite elements," IEEE Transactions on Ultrasonics, Ferroelectrics, and Frequency Control, vol. 54, no. 10, pp. 2045-2051, 2007.

[8] L. Gavrić, "Computation of propagative waves in free rail using a finite element technique," Journal of Sound and Vibration, vol. 185, no. 3, pp. 531-543, 1995.

[9] W. Li, R. A. Dwight, and T. Zhang, "On the study of vibration of a supported railway rail using the semi-analytical finite element method," Journal of Sound and Vibration, vol. 345, pp. 121-145, 2015.

[10] E. Tassilly, "Propagation of bending waves in a periodic beam," International Journal of Engineering Science, vol. 25, no. 1, pp. 85-94, 1987.

[11] P. Wang, Q. Yi, C. Zhao, and M. Xing, "Elastic wave propagation characteristics of periodic track structure in highspeed railway," Journal of Vibration and Control, vol. 25, no. 3, pp. 517-528, 2019.

[12] M. Oregui, Z. Li, and R. Dollevoet, "An investigation into the modeling of railway fastening," International Journal of Mechanical Sciences, vol. 92, pp. 1-11, 2015. 
[13] S. L. Grassie, "Rail corrugation: characteristics, causes, and treatments," Proceedings of the Institution of Mechanical Engineers - Part F: Journal of Rail and Rapid Transit, vol. 223, no. 6, pp. 581-596, 2009.

[14] M. S. Kushwaha, P. Halevi, L. Dobrzynski, and B. DjafariRouhani, "Acoustic band structure of periodic elastic composites," Physical Review Letters, vol. 71, no. 13, pp. 2022-2025, 1993.

[15] X. Sheng, C.-Y. Zhao, Q. Yi, P. Wang, and M.-T. Xing, "Engineered metabarrier as shield from longitudinal waves: band gap properties and optimization mechanisms," Journal of Zhejiang University - Science, vol. 19, no. 9, pp. 663-675, 2018.

[16] P. Wang, Q. Yi, C. Zhao, M. Xing, and J. Tang, "Wave propagation in periodic track structures: band-gap behaviours and formation mechanisms," Archive of Applied Mechanics, vol. 87, no. 3, pp. 503-519, 2017.

[17] M. Oregui, Z. Li, and R. Dollevoet, "An investigation into the vertical dynamics of tracks with monoblock sleepers with a $3 \mathrm{D}$ finite-element model," Proceedings of the Institution of $\mathrm{Me}$ chanical Engineers - Part F: Journal of Rail and Rapid Transit, vol. 230, no. 3, pp. 891-908, 2016.

[18] A. C. Eringen and E. S. Suhubi, Elastodynamics, Volume II: Linear Theory, Academic Press, New York, NY, USA, 1975.

[19] Y.-F. Wang, Y.-S. Wang, and X.-X. Su, "Large bandgaps of two-dimensional phononic crystals with cross-like holes," Journal of Applied Physics, vol. 110, no. 11, p. 113520, 2011. 